\title{
Nanofluidization electrostatics
}

\author{
J. M. Valverde, ${ }^{1}$ M. A. S. Quintanilla, ${ }^{1}$ M. J. Espin,,${ }^{1,2}$ and A. Castellanos ${ }^{1}$ \\ ${ }^{1}$ Faculty of Physics, University of Seville, Avenida Reina Mercedes s/ $n, 41012$ Seville, Spain \\ ${ }^{2}$ Department of Applied Physics II. EUAT.University of Seville, Avenida Reina Mercedes 4A, 41012 Seville, Spain
}

(Received 21 December 2007; published 12 March 2008)

\begin{abstract}
Electrostatic charging of powders is a relevant phenomenon for a number of industrial applications. The design of new processes and the use of high resistivity materials and ultrafine powders may lead to higher charging rates and to higher levels of charge accumulation that can become a serious problem. In this work we investigate experimentally electrostatic charging in nanofluidization. The behavior of a fluidized bed of silica nanoparticles under the influence of an electrostatic field is studied. The electric field is applied in the horizontal direction and perpendicular to the gas flow. On one hand, we observe the influence of the electric field on the bulk behavior of the fluidized bed, which suffers a collapse when the electric field is turned on. For strong electric fields the stationary state of the fluidized bed reminds one of that of a spouted bed, with a solid layer adhered to the wall and a low density core region of local high gas velocity. On the other hand, and in order to gain additional insight, we look at the trajectories of nanoparticle agglomerates as affected by the electric field. This images analysis reveals that these agglomerates are horizontally deflected towards the wall as a consequence of being charged. From the analysis of agglomerate trajectories the charge per agglomerate is estimated. Using these measurements the electrostatic forces between agglomerates are calculated and compared to van der Waals attractive forces.
\end{abstract}

DOI: 10.1103/PhysRevE.77.031301

PACS number(s): 45.70. $-\mathrm{n}, 4$ 47.55.Lm, 41.20.Cv

\section{INTRODUCTION}

Flowing powders can be quite electrostatically active. During the handling of powders, electrostatic charging usually occurs and it is attributed to the processes of contact and triboelectric charging [1-4]. Electrostatic energy stored in large quantities of powders can be large enough to cause sparks to be able to ignite the material. Reports of the occurrence of major dust explosions due to electric sparks and static discharges date back to the 18th century [3]. A particularly hazardous process is the pneumatic transport of powder in which, even though the powder is dispersed in air, high charge levels are often generated that give rise to static discharges when the powder settles $[3,4]$. Other nuisance situations related to electrostatics are sticking of powders to containers or the collection of dust by fabrics [1]. Electrostatic charge may also affect powder handling operations due to enhanced interparticle attractive forces [4]. The opposite problem of particle repulsion also exists, leading to difficulties in powder delivery [4]. However, electrostatic forces in powders can be effectively used as well in many industrial applications such as xerographic printers [5], pollution control [6], electrostatic separators $[7,8]$ and coating in fluidized beds [7] to name a few. The physics of these processes is not completely understood and depends upon many parameters, which are often uncontrolled in industrial situations [4]. Recently, the development of new industry processes demanding high charge and specific surface levels has created an interest in the production and charging of nanometric particles with extremely high surface-charge density [9].

Fluidization of nanoparticles, providing extremely high fluid-solid contact and reaction efficiency, has become one of the most promising techniques for emerging industrial applications. Fluidization is accomplished by passing gas uniformly through the powder by a porous supporting plate. At a certain gas flow the drag balances the powder weight per unit area and the powder becomes fluidized. Recent reports of uniform fluidization of moderate density nanoparticles [10-20] have coined the word "nanofluidization" to denote this type of fluidization of nanometric particles also known as ultrafine particles. Even in the case of some nanoparticles [11] full suppression of bubbling has been observed, i.e., the fluidized bed transits directly from the uniform fluidlike regime to elutriation of the nanoparticles at high gas velocities [14]. The suppression of gas bubbles in fluidized beds of nanoparticles for gas velocities well above the minimum fluidization velocity is related to the existence of porous light agglomerates. Many works have dealt with the problem of estimating the size of nanoparticle agglomerates (see Ref. [21] for a review). Noninvasive laser-based planar imaging has been developed in recent years. In this technique in situ images of the fluidized agglomerates are obtained with the aid of a laser source focused on the fluidized bed surface $[10-12,14,16,18,19]$. Photographs of agglomerates in the splash zone of the fluidized bed show an average size of the order of hundreds of microns and a broad size distribution. Wang et al. [13] have recently used particle image velocimetry analysis and laser-based planar imaging to measure the terminal settling velocity and size of single agglomerates. By relating both measurements they have obtained that most of the agglomerates have a fractal dimension close to $D_{a}$ $=\ln N_{a} / \ln k_{a}=2.5$, suggesting a predominance of the diffusion limit agglomeration (DLA) type. Here $N_{a}$ is the average number of primary nanoparticles in an agglomerate and $k_{a}$ is the average ratio of agglomerate size $d^{* *}$ to primary nanoparticle size $d_{p}$.

While there is a general consensus on the size of nanoparticle agglomerates in fluidization [21], the research on nanoparticle charging in fluidized beds is still scarce. Kashyap et al. [22] have investigated the effect of a horizontal electric field on a bed of $10 \mathrm{~nm}$ silica nanoparticles uni- 
formly fluidized by air. A main observation reported in this recent work is that the electric field markedly hindered bed expansion. The authors explain the experimental results as due to the addition of a vertical electrical force to the gravity force. By inclusion of this electrostatic force ad hoc in a one-dimensional momentum balance equation, their model further predicted a decrease of the agglomerate size and granular temperature of the nanoparticles as the strength of the electric field is increased. In the present work we report similar experimental observations on the bulk behavior of the fluidized bed as affected by the application of a horizontal electrostatic field. Our relevant contribution consists of the direct visualization of the dynamics of fluidized agglomerates by using high speed video analysis. These experiments allow us to provide a clear explanation to the observed decrease of bed expansion upon application of the electric field. Moreover, these measurements enable us to report an estimation of agglomerate charge, which might be valuable information in order to predict possible hazards in potential applications.

In the following subsections we give a brief review on the fundamentals of particle charging in powders. Section III is devoted to a description of the experimental setup. In Sec. IV the experimental results are presented and discussed. Finally, we outline the conclusions of this work in Sec. V.

\section{A. Measurements of particle charge in fine powders}

The electric force acting on a suspended particle of net charge $Q$ is given by

$$
\mathbf{F}=Q \mathbf{E}+(\mathbf{p} \cdot \nabla) \mathbf{E},
$$

where $Q$ is the net charge on the particle, $\mathbf{p}$ is the dipole moment of the particle, and $\mathbf{E}$ is the electric field vector. If the net charge of the particle is close to zero the right-hand side of Eq. (1), known as the dielectrophoretic force, will dominate. In general, dielectrophoresis is an effect requiring quite divergent fields and usually requires sizable differences in the permittivities of the particle and the surrounding medium [23]. For charged particles in an electrostatic field the direct attraction of the electric field for a charge (electrophoretic force) is dominant, even when relatively small charges are present.

The first experiment to measure the charge of blown fine powder particles had occurred to Sachsse in 1932 [24] by using the Millikan oil drop method. Sachsse obtained that particles acquired charges ranging from $10^{-17}$ to $10^{-15} \mathrm{C}$, depending on the type of material and method of dust generation. In 1950 Kunkel and Hansen [25] described an improved device, inspired in the Hopper and Laby modification of the oil drop experiment, to measure the size and charge of blown powder particles. The horizontal deflection of particles as they settle under the influence of gravity in a horizontal electric field was recorded photographically by dark field illumination periodically interrupted by a rotating shutter. Then, the size and charge of the particles were derived by the application of Stokes' law. Results from this experiment were presented by Kunkel in a separate paper [26]. Particles of diverse materials in sizes ranging from 0.5 to $30 \mu \mathrm{m}$ were analyzed. The charges measured varied from 0 to $10^{-14} \mathrm{C}$ depending on the type of material and on the dispersion process employed. Generally, it was observed that all dusts contained charged particles. These included electroneutral homogeneous dusts gently dispersed and with no impact on solid surfaces, for which the charges of opposite sign measured were about the same.

Current instruments for measuring the charge of powder particles from measurements of electrical mobility distributions are based on those pioneer works [27-29]. In electrostatic elutriators and spectrometers the aerosol flows through a flat channel in which a transversal electric field is applied and particles are removed by deposition onto the wall due to electrophoretic forces. Other methods consist of measuring the electrical potential or current due to collected charged particles by some devices [27]. For example, the electrical current in a Faraday cage induced by the charged particles has served to measure the charge of aerosol particles [29]. Measurements of the potential of a capacitor connected between ground and a metal filter holder in which the charged blown powder is accumulated have shown that powders such as starch or Cabosil ${ }^{\odot}$ may have up to $10^{-3} \mathrm{C}$ of charge per kilogram of powder [1].

The charging tendency of dielectric powders measured in industrial operations show little dependence on the materials themselves but are rather dependent on the type of operations and the specific surface area of the powders [3]. For instance, it has been observed that sieving can charge the powder in a range between $10^{-11} \mathrm{C} / \mathrm{kg}$ and $10^{-9} \mathrm{C} / \mathrm{kg}$, and pouring in the range $10^{-9}-10^{-7} \mathrm{C} / \mathrm{kg}$. On the other hand, operations involving high energy input such as micronizing or pneumatic transfer create charge levels in the range $10^{-7}-10^{-4} \mathrm{C} / \mathrm{kg}[2-4]$. In general, the greater the energy of operation and specific surface area of the powder the greater is the measured charging $[2,3]$. Experimental values for the charge-to-mass ratio $Q m r$ of many powders of different specific $S$ surface area obtained using laboratory scale pneumatic transport devices are reviewed in Ref. [3]. It is shown that the rate of increase of $Q m r$ with $S$ is more or less linear $\left(Q m r \sim 10^{-7} \mathrm{~S}\right.$, where $Q m r$ is expressed in $\mathrm{C} / \mathrm{kg}$ and $S$ is in the range between $1 \mathrm{~m}^{2} / \mathrm{kg}$ and $10^{3} \mathrm{~m}^{2} / \mathrm{kg}$ ) [3].

The maximum surface charge that can be attained by a particle usually depends on the breakdown field strength $E_{\max }$ of air. For a charged flat surface in air, the expected breakdown threshold is $E_{\max } \sim 3 \times 10^{6} \mathrm{~V} / \mathrm{m}$, which corresponds to a surface charge density $\sigma \sim \epsilon_{0} E_{\max }$ $\simeq 26.5 \mu \mathrm{C} / \mathrm{m}^{2}[4,8]$. In the case of a curved surface, and due to the high nonuniformity of the electric field near the surface, higher fields can be sustained. An empirical formula for the maximum field that can be supported in terms of the particle diameter $d_{p}$ of a spherical particle is $E_{\max }$ $\sim 10^{6} / d_{p}^{0.3} \mathrm{~V} / \mathrm{m}$ ( $d_{p}$ in meters), which gives a maximum charge per particle $Q_{\max } \sim 3 \times 10^{-5} d_{p}^{1.7} \mathrm{C}$ and a charge-tomass ratio $Q m r \sim 6 \times 10^{-5} /\left(\rho_{p} d_{p}^{1.3}\right) \mathrm{C} / \mathrm{kg}$ [4]. For example, for 50- $\mu \mathrm{m}$-sized particles it is obtained $Q_{\max } \sim 10^{-12} \mathrm{C}$ and $Q m r \sim 0.02 \mathrm{C} / \mathrm{kg}$ (using $\rho_{p}=2500 \mathrm{~kg} / \mathrm{m}^{3}$ ). It is, however, uncertain whether this equation might apply to smaller particles that are usually aggregated due to the predominance of interparticle attractive forces over particle weight [30]. 


\section{B. Mechanism of particle charging in fine powders}

Why powders charge has been the subject of considerable research, yet truly quantitative and repeatable results are difficult to obtain $[1,27]$. Commonly, the mechanism of charging is regarded as a result of collisions between particles and possibly friction of very short duration (triboelectric charging). However, most investigations suggest that the main cause for the charge buildup on powders during industrial handling and processing is contact charging [3]. According to this mechanism, the main charge separation occurs at the first mechanical separation of the particles due to the dissimilarity between the work functions of the materials at contact. Generally, electrification is governed by the physics of the contact between the surfaces separated. Nevertheless, shearing forces during friction may change the work functions of the materials, which might affect the charging tendency [3].

In his pioneer work Kunkel [26] had already suggested that powder charging should occur at mechanical separation of contacting surfaces at the moment of dispersion. Contact charging would explain why the dust cloud was electroneutral as a whole, but with almost all the particles being charged. Kunkel [26] pointed out that transfers of electrons on particle separation were easily possible due to imperfections at the contact area. He proposed a theory of random charge transfer by which particle charge was predicted to increase with particle diameter as measured experimentally [26]. Loeb [31] claimed in accordance that, in most cases, charge transfer required only intimate contact, while for two identical surfaces the frictional charging effect was rare. Currently, it is generally accepted that charges are exchanged whenever any two surfaces come into contact with each other even for cases where the bulk materials are the same due to surface impurities and imperfections [32]. If contact is broken, a net charge may subsequently exist on each of the surfaces. Yet, the electrification at interfaces and the charges remaining after the surfaces in contact are separated are completely unpredictable due to the effect of numerous uncontrolled parameters such as surface imperfections and contaminants, ambient humidity, nature of the contact, speed of separation, charge carrier species, and charge back flow across the interface $[3,32,33]$. In general, the work functions are not well defined for most materials, particularly for those which are amorphous [34].

\section{ROLE OF PARTICLE CHARGING ON POWDER FLOWABILITY}

In situations in which particles carry a substantial charge level, electrostatic interactions could play a role on the rheological behavior of powders $[35,36]$. Theoretically, the electrostatic force between two charged dielectric spheres of size $d_{p}$ in contact, carrying charges $Q_{1}$ and $Q_{2}$ and immersed in a dielectric surrounding medium of permittivity $\epsilon_{m}$, can be written in the form [35]

$$
f_{e}=\frac{Q_{2}^{2}}{4 \pi \epsilon_{m} d_{p}^{2}}\left\{\alpha\left[\left(\frac{Q_{1}}{Q_{2}}\right)^{2}+1\right]-\beta \frac{Q_{1}}{Q_{2}}\right\} .
$$

In our case the ambient fluid is a gas, thus $\epsilon_{m} \simeq \epsilon_{0}$. The first term on the right side of Eq. (2) represents the attraction from the image charge of $Q_{1}$ induced in the sphere 2 . The second term describes the net dielectrophoretic force on the sphere 1 induced by the nonhomogeneous electric field due to the charge on sphere 2 . The third term is due to the Coulomb force between both spheres. Feng [35] has used the Galerkin finite-element method to investigate the dependence of the coefficients $\alpha$ and $\beta$ on the permittivity $\epsilon$ of the spheres. For instance, one would obtain $\alpha \simeq 0.25$ and $\beta$ $\simeq 1.3$ for $\epsilon=3.8 \epsilon_{0}$, corresponding to amorphous silica [37]. Thus, for $50 \mu \mathrm{m}$ silica spheres charged to the levels measured in pneumatic transfer $\left(Q m r \sim 10^{-4} \mathrm{C} / \mathrm{kg}, Q \sim 1.6\right.$ $\times 10^{-14} \mathrm{C}$ ) [2], the electrostatic force is $f_{e} \simeq 1.7 \mathrm{nN}$ (attractive) for charges of different sign and $f_{e} \sim-0.8 \mathrm{nN}$ (repulsive) for charges of equal sign. These values are comparable to particle weight $w_{p} \sim 1.6 \mathrm{nN}$ (using $\rho_{p}=2500 \mathrm{~kg} / \mathrm{m}^{3}$ ).

It must be noted that the above calculations are made for ideally spherical particles. Hays [38] has pointed out that the electrostatic adhesion force for irregularly shaped particles can be larger than that calculated from Eq. (2) due to nonuniform charge distribution, albeit quantitative predictions are difficult due to the unpredictability of the charged areas really in contact. According to Hays [38], the electrostatic adhesion between an irregularly shaped particle and a flat conducting substrate can be approximated by

$$
f_{H}=Q f \frac{\sigma}{2 \epsilon_{0}},
$$

where $\sigma=Q / A_{t}$ is the surface-charge density, $A_{t}$ is the total area of the charge patches of the particle, and $f=A_{c} / A_{t}$ is the ratio of the sum of the charged areas in contact with the conducting surface $A_{c}$ to $A_{t}$. In the cascade development process, widely used in commercial systems for copying documents [34], $\sim 10 \mu \mathrm{m}$ toner particles are charged when mixed with $\sim 500 \mu \mathrm{m}$ carrier particles of different material. Measurements on standard carrier-toner combinations give a particle charge between $10^{-12}$ and $10^{-14} \mathrm{C}$ for carrier beads, and between $10^{-14}$ and $10^{-16} \mathrm{C}$ for toner particles [34,38]. In order to illustrate his theory, Hays [38] used as typical values for tribo-charged xerographic toner particles $f=0.2, Q$ $=10^{-14} \mathrm{C}$, and $\sigma=10^{-3} \mathrm{C} / \mathrm{m}^{2}$, and estimated an electrostatic force of adhesion $f_{H}=100 \mathrm{nN}$, which is one order of magnitude higher than that expected for perfectly spherical particles.

Besides the electrostatic force we must consider the ubiquitous van der Waals attractive force produced by the interaction of fluctuating molecular dipole fields [39]. Assuming that retardation effects are negligible and that the interaction between molecules is pairwise, Hamaker [40] summed up all these interactions between two spherical and rigid particles at contact. The van der Waals attractive force between two spherical particles of same size $d_{p}$ at contact could be approximated by

$$
f_{v d W} \simeq \frac{A d_{p}}{24 z_{0}^{2}}
$$

where $A$ is the Hamaker constant (for most solids $A$ $\simeq 10^{-19} \mathrm{~J}[41]$ ), and $z_{0} \simeq 3-4 \AA$ is the minimum intermolecular distance [42]. Because of the short range of the mo- 

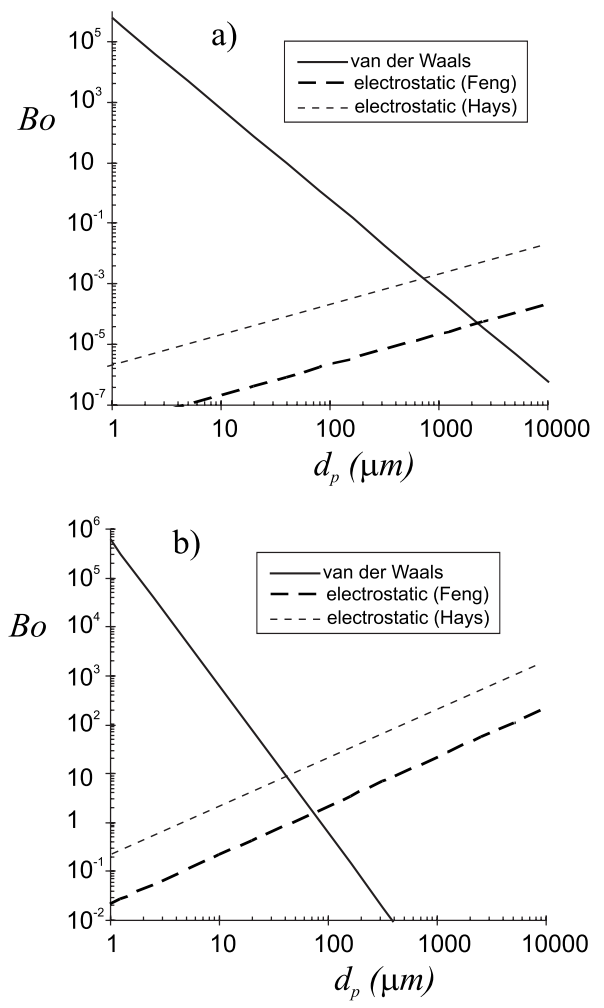

FIG. 1. Ratio of interparticle attractive force to particle weight as a function of particle size. As indicated interparticle forces are calculated according to the van der Waals interaction (solid lines) and electrostatic interaction (dashed lines). Results are shown for silicon dioxide $\left(\epsilon=3.8 \epsilon_{0}, \alpha \simeq 0.25, \beta \simeq 1.3, \rho_{p}=2500 \mathrm{~kg} / \mathrm{m}^{3}\right)$. In (a) the electrostatic force is calculated from the typical charge-to-mass ratio measured in pouring processes. In (b) the electrostatic force is calculated from the typical charge-to-mass ratio measured in pneumatic transfer processes. Only the attractive electrostatic force is calculated. There will be also a repulsive electrostatic force of the same order of magnitude between charges of the same sign.

lecular interaction, the van der Waals force is actually determined by the local radius of curvature of the surface asperities at contact. Therefore, for micrometric and larger particles, the typical size of the surface asperities $d_{a}$ must be used in Eq. (4) instead of the particle diameters. A typical value reported for the size of surface asperities of powder particles is $d_{a} \simeq 0.2 \mu \mathrm{m}$ [42-45]. Thus, we can estimate $f_{v d W} \sim 10 \mathrm{nN}$ (attractive), independently of particle size.

Figure 1 serves as an illustration of the relative importance of electrostatic and van der Waals forces on powder flowability depending on particle size. In this figure the ratios $f_{e} / w_{p}$ and $f_{v d W} / w_{p}$ are plotted as a function of particle size $d_{p}$. The ratio of interparticle force to particle weight is usually defined as the granular Bond number (Bo) and is a measure of powder cohesiveness. For Bo $\lesssim 1$ the material shows a free-flowing behavior whereas for $\mathrm{Bo} \gtrsim 1$ the particles have a tendency to aggregate that inhibits powder flow [46]. In the calculations we have used typical values for silica $\left(\epsilon=3.8 \epsilon_{0}, \alpha \simeq 0.25, \beta \simeq 1.3, \rho_{p}=2500 \mathrm{~kg} / \mathrm{m}^{3}\right)$. Figure 1(a) shows that the electrostatic force estimated from the charge level typically measured in a low energy process such as pouring $\left(Q m r \sim 10^{-7} \mathrm{C} / \mathrm{kg}\right)$ does not play a role practi- cally in the whole range of particle sizes. On the other hand, it is seen in Fig. 1(b) that, if we use the charge level typically measured in a high energy process such as pneumatic transfer $\left(Q m r \sim 10^{-4} \mathrm{C} / \mathrm{kg}\right)$, the hindrance of granular flow due to the attractive electrostatic force can be relevant for $d_{p}$ $\gtrsim 200 \mu \mathrm{m}$. Thus, high charge levels of coarse grains could indeed inhibit the flow of otherwise free-flowing materials. If we assume, according to Hays [38], that the irregular morphology of the particle surface produces an increase of one order of magnitude of the electrostatic force over the entire range of particle sizes, the relevance of this force on flow hindrance would be shifted to smaller sizes. By inspection of Fig. 1 it can be estimated that, in the case of high charge levels, the electrostatic force would become significant for $d_{p} \gtrsim 50 \mu \mathrm{m}$ for silica particles. In any case, it is clear from these figures that the van der Waals force is the dominant interaction between blown fine powder particles.

\section{EXPERIMENTAL SETUP AND PROCEDURE}

The fluidized bed setup used in the present work consists of a vertical $2.54 \mathrm{~cm}$ internal diameter polycarbonate vessel, $16.2 \mathrm{~cm}$ in height, which is fitted at the bottom with a sintered stainless steel plate having a pore size of $5 \mu \mathrm{m}$ that acts as gas distributor. The fluidization cell is fixed with a teflon holder and is placed between two parallel square electrodes of $14 \mathrm{~cm}$ of side length. The typical distance between the electrodes is $d=7.6 \mathrm{~cm}$. One of the electrodes is grounded and high voltage $V$ (up to $30 \mathrm{KV}$ ) is applied to the opposite electrode using a high voltage dc supply (Spellman model HRH 40PN120/OVP/FG with a range of 0-40 KV).

Ideally, the electric field strength is pretended to be uniform within the fluidized bed. However, and due to the presence of the metallic gas distributor, the electric field could be distorted near the base of the cell. Other possible sources of field heterogeneity are the finite size of the electrodes and the presence of the dielectric walls of the fluidization cell. To evaluate the spatial extension of these perturbations we have carried out 2D and 3D FEM calculations of the electric field between the electrodes using the software CONSOL. The electric field and equipotential lines calculated showed that these perturbations are not significant within the bed. According to these calculations the electric field strength within the bed can be taken as approximately constant $(E \simeq V / d)$.

The material used in the experiments was silica Aerosil@R974 from Degussa Company (particle size $12 \mathrm{~nm}$, particle density $2250 \mathrm{~kg} / \mathrm{cm}^{3}$ ). The powder was presieved (using a sieve opening of $500 \mu \mathrm{m}$ ) in order to remove previously existing very large agglomerates. In order to correct for elutriation, the presieved powder was weighed before and after each fluidization run. The mass of the sample to be fluidized $m$ was in the range between 0.20 and $0.45 \mathrm{~g}$.

Previous reports $[10-12,14]$ show that when subjected to a gas flow, silica nanopowder exhibits agglomerate particulate fluidization (APF) behavior, characterized by a large bed expansion and apparent smooth fluidization. Typical values of agglomerate size for this powder in the fluidized bed measured by several authors using noninvasive techniques are of the order of hundreds of microns (see Ref. [21] for a review). 
In the present work we have concentrated on the effect of application of an electric field on the expansion of the uniformly fluidized bed. Once the sample was presieved, weighed, and placed in the cell, it was initialized by applying a sufficiently high gas flow. The bed height was measured when it reached a stationary state, starting from a gas flow rate of $1000 \mathrm{~cm}^{3} / \mathrm{min}$, and subsequently decreasing the gas flow rate in decrements of $100 \mathrm{~cm}^{3} / \mathrm{min}$. In a second step, the sample was initialized again and the electric field was applied after the fluidized bed had reached the stationary state. Then the fluidized bed height, with the electric field turned on, was again measured as the gas flow rate was slowly decreased. This procedure was followed for several values of the electric field strength. The flow of gas (dry nitrogen) to the column was controlled by means of a MKS flow controller (model 1179A) with a full range from 0 to $2000 \mathrm{~cm}^{3} / \mathrm{min}$. The height of the fluidized bed was measured using a ruler taped to the side of the bed (marks in $\mathrm{mm})$.

Additionally, local observations were performed that allowed us to gain insight into the agglomerate dynamics upon application of the electric field. Images of the fluidized bed were acquired at 462 frames per second using a CMOS highspeed camera VDS Vosskühler HCC-1000 mounting a Cosmicar/Pentax optics with $16 \mathrm{~mm}$ focal length and F1.4 aperture, and a $1 \mathrm{~mm}$ extension tube to increase the magnification of the optics. The camera was separated about $4 \mathrm{~cm}$ from the gap between the electrodes to avoid interaction with the electric field. Illumination was provided by a $90 \mathrm{~W}$ halogen light source Intralux 4000-1 (Volpi AG). The external diameter of the fluidization cell $(3.18 \mathrm{~cm})$ was used for spatial calibration of the images. The image size was 1024 $\times 512$ pixels grayscale (256 levels), with the larger dimension of the image aligned with the axis of the fluidization cell, thus yielding a maximum resolution of $49.6 \mu \mathrm{m}$ per pixel. In order to observe the effect of the electric field, the acquisition of each sequence of images was started before the electric field was turned on.

\section{EXPERIMENTAL RESULTS AND DISCUSSION}

Figure 2 shows experimental data on the particle volume fraction $\phi$ of the fluidized bed as a function of the superficial gas velocity $v_{g}$. The particle volume fraction $\phi$ is calculated from the measured height $h$ of the bed $\left[\phi=m /\left(\rho_{p} A h\right)\right.$, where $A=5 \mathrm{~cm}^{2}$ is the bed cross-sectional area]. In order to look for data reproducibility, each point of these curves represents the average and standard deviation over 36 independent runs (for zero electric field) and 12 independent runs (for each value of the strength of the electric field). As can be seen in this figure, the main effect of the application of the electric field is an apparent increase of the particle volume fraction of the fluidized bed. This observation agrees qualitatively with the results recently reported by Kashyap et al. using a similar setup [22]. In our experiments we observed that the effect of the electric field on bed expansion was not reversible: when the electric field was turned off, the height of the bed did not return to its original value in the absence of field (see Fig. 3). This observation suggests that the electric field must have a

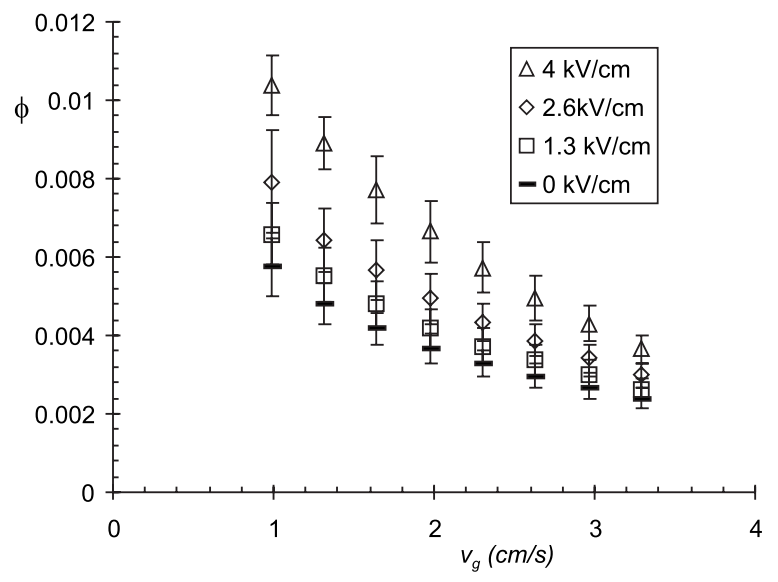

FIG. 2. Average particle volume fraction of the fluidized bed $\phi$ as a function of the superficial gas velocity $v_{g}$ for different strengths of the electric field applied.

relevant influence on bed dynamics, possibly consisting of irreversible solid compaction of the powder due to electrical forces on the agglomerates. Clearly, direct visualization of the bed dynamics at a local scale would be of great importance in order to gain physical insight into the mechanisms by which the electric field influences fluidization.

\section{A. Effect of the electric field on agglomeration}

When the nanoparticle bed is fluidized, preexisting simple agglomerates build up the complex agglomerates in a dynamic process $[15,16]$. In this self-rearranging process of simple agglomerates, a stationary state is reached which is characterized by an average size $d^{* *}$ of the complex agglomerates. Most of the simple agglomerates observed by Yao et $a l$. have a size $d^{*}$ between 1 and $100 \mu \mathrm{m}$. The work of Nam et al. [10] suggests $d^{*} \approx 30 \mu \mathrm{m}[30]$. The size of the complex agglomerates has been predicted from the balance between the drag force on a simple agglomerate attached to the

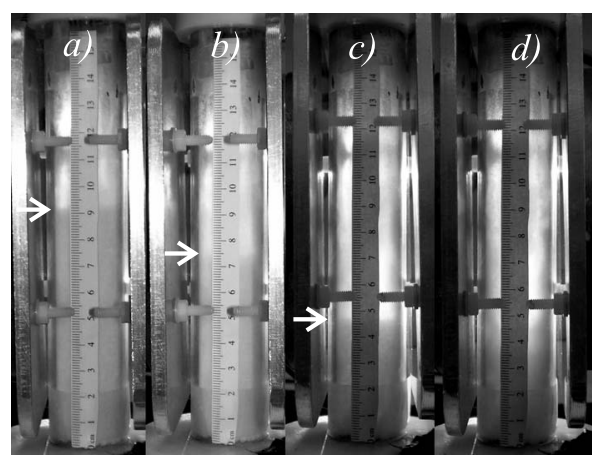

FIG. 3. Series of photographs showing the irreversibility of the effect of the electric field on fluidized bed expansion. The superficial gas velocity is $2.6 \mathrm{~cm} / \mathrm{s}$. The strength of the electric field $E$ is slowly increased from (a) to (c). (a) $E=0 \mathrm{~V} / \mathrm{m}$. (b) $E=0.66$ $\times 10^{5} \mathrm{~V} / \mathrm{m}$. (c) $E=1.32 \times 10^{5} \mathrm{~V} / \mathrm{m}$. In (d) the field is turned off. The arrows indicate the evolution of the free surface. In (d) the free surface cannot be neatly distinguished. 


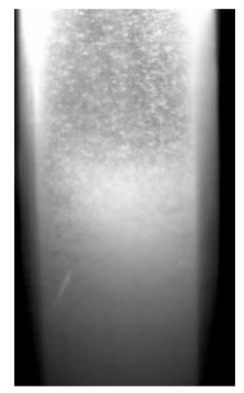

a) $\mathrm{t}=0 \mathrm{~ms} \mathrm{E}=0 \mathrm{~V} / \mathrm{m}$

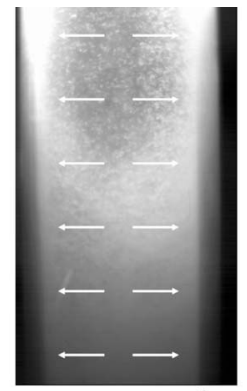

b) $\mathrm{t}=106 \mathrm{~ms} \mathrm{E}=2 \times 10^{5} \mathrm{~V} / \mathrm{m}$

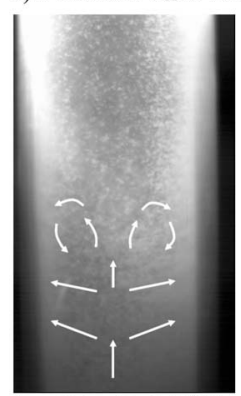

e) $\mathrm{t}=539 \mathrm{~ms} \mathrm{E}=2 \times 10^{5} \mathrm{~V} / \mathrm{m}$

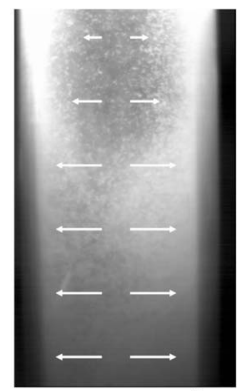

c) $\mathrm{t}=214 \mathrm{~ms} \mathrm{E}=2 \times 10^{5} \mathrm{~V} / \mathrm{m}$

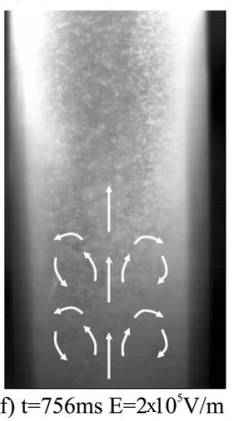

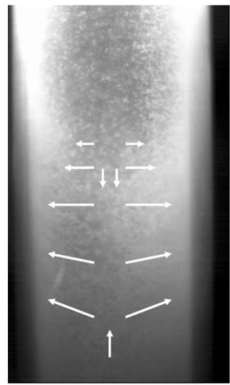

d) $t=323 \mathrm{~ms} \mathrm{E}=2 \times 10^{5} \mathrm{~V} / \mathrm{m}$

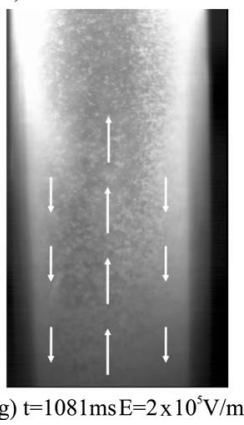

FIG. 4. Snapshots of the fluidized bed near the free surface showing the main qualitative features of the effect of the electric field on the flow field at different times since it was applied. In (a) there is no applied field. From (b) to (g) the strength of the applied horizontal field is $E=2.5$ $\times 10^{5} \mathrm{~V} / \mathrm{m}$. The arrows show the main agglomerate flow lines. Video of the experiment is available on request. complex agglomerate and the adhesion force $f^{*}$ between simple agglomerates [21]. In the absence of humidity the main contribution to $f^{*}$ is the van der Waals force $\left(f_{v d W}^{*}\right.$ $\sim 10 \mathrm{nN}$ ), which enables us to obtain a value of $d^{* *}$ of the order of hundreds of microns, in agreement with experimental observations [21].

Although it is likely that these agglomerates are charged, it is reasonable from our previous analysis to neglect the electrostatic force as compared to van der Waals force. On the other hand, these agglomerates can be polarized when the electric field is applied, giving rise to an additional force that still needs to be discussed. This force between induced dipoles might lead to chainlike structures as seen in many kinds of electrorheological fluids containing dielectric particles $[47,48]$. It is well known from fundamental electrostatics that the dipole moment induced by the electric field on a spherical dielectric particle of permittivity $\epsilon$ in a surrounding medium of permittivity $\epsilon_{0}$ can be calculated from [49]

$$
p=\frac{1}{6} \pi d_{p}^{3} 3 \epsilon_{0} \frac{\epsilon-\epsilon_{0}}{\epsilon+2 \epsilon_{0}} E .
$$

The force between these interacting dipoles at contact is given by

$$
f_{d}=\frac{3 p^{2}}{2 \pi \epsilon_{0} d_{p}^{4}} .
$$

Using as typical values in our experiment $d_{p}=12 \mathrm{~nm}, \epsilon_{0}$ $=8.854 \times 10^{-12} \mathrm{~F} / \mathrm{m}, \epsilon=3.8 \epsilon_{0}$, and $E=4 \times 10^{5} \mathrm{~V} / \mathrm{m}$, it is obtained $f_{d} \sim 10^{-27} \mathrm{~N}$ for the force between the polarized primary nanoparticles. This negligible value as compared to the interparticle van der Waals force $\left[f_{v d W} \simeq A d_{p} /\left(24 z^{2}\right)\right.$ $\simeq 0.5 \mathrm{nN}]$ indicates that the electric field cannot modify directly the internal structure of the agglomerates.

The force between simple agglomerates $f_{d}^{*}$ due to their polarization by the electric field can be estimated using the above equations if these aggregates are considered as effec- tive dielectric spheres of size $d^{*}$. According to effective medium theories for aggregates and numerical analysis on random dielectric materials [50], the effective permittivity $\epsilon^{*}$ of an agglomerate of dielectric spherical particles of permittivity $\epsilon$ located in a homogeneous environment of permittivity $\epsilon_{0}$ can be calculated from the Bruggeman mixing rule

$$
\left(1-\phi_{i n t}\right) \frac{\epsilon_{0}-\epsilon^{*}}{\epsilon_{0}+2 \epsilon^{*}}+\phi_{i n t} \frac{\epsilon-\epsilon^{*}}{\epsilon+2 \epsilon^{*}}=0,
$$

where $\phi_{\text {int }}$ is the particle volume fraction within the agglomerate $\left[\phi_{\text {int }}=\left(d^{*} / d_{p}\right)^{D_{a}-3}\right]$. Using as typical values $d^{*}$ $=30 \mu \mathrm{m}$ and $D_{a}=2.5$, it is obtained $\epsilon^{*} \simeq 1.03 \epsilon_{0}$ and, from the modified versions of Eqs. (5) and (6), $f_{d}^{*} \sim 6 \times 10^{-24} \mathrm{~N}$, which denies any effect of polarization on the dynamics of aggregation between simple agglomerates. Finally, if complex agglomerates are considered as effective spherical particles of size $d^{* *} \simeq 200 \mu \mathrm{m}$, the force between complex agglomerates due to the dipole moment induced by the electric field can be estimated as $f_{d}^{* *} \sim 4 \times 10^{-23} \mathrm{~N}\left(\epsilon^{* *} \simeq 1.01 \epsilon_{0}\right)$. Thus, it must be concluded that the polarization induced by the electric field does not affect essentially the attractive forces between agglomerates in the fluidized bed and, therefore, has no direct effect on agglomeration.

\section{B. Effect of the electric field on the flow dynamics}

To obtain insight into the physics of the fluidized bed as affected by the external field, direct visualization of the agglomerate flow dynamics has been carried out using a fast camera as detailed in the description of the experimental setup. Figure 4 shows snapshots of the fluidized bed near the splash zone that illustrate the main qualitative features of the flow dynamics evolution after application of the external field. The arrows indicate the preferential lines of the flow of agglomerates in each situation. In the absence of the external electric field [Fig. 4(a)] the agglomerates are seen to display 


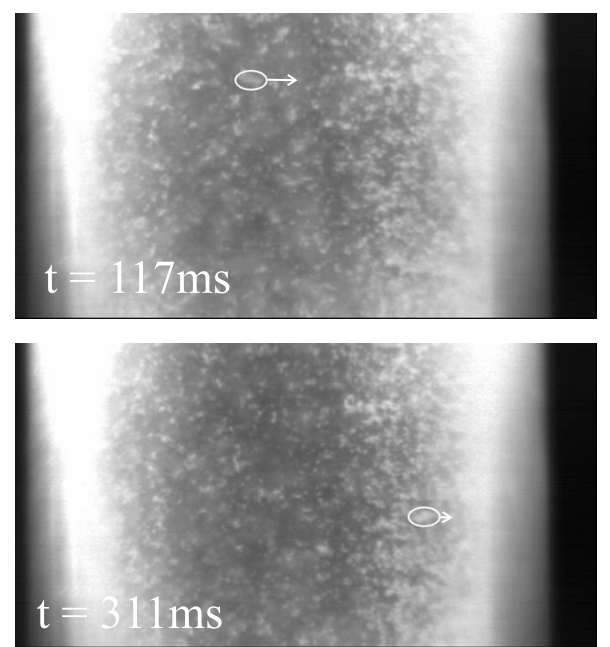

FIG. 5. Snapshots of the fluidized bed near the free surface showing the deflection of an agglomerate towards the wall. The time elapsed since application of the electric field is indicated. The strength of the applied horizontal field is $E=2.5 \times 10^{5} \mathrm{~V} / \mathrm{m}$. Video of the experiment is available on request.

a pseudoturbulent random motion with the smaller agglomerates being elutriated off the bed.

As schematized in Fig. 4(b), a relevant phenomenon, just when the horizontal electric field is turned on, is a preferential flow of agglomerates in the direction of the field and towards the walls. The analysis of the trajectories of the agglomerates as deflected by the electric field will serve us in the next section to have an estimation of the charge of the agglomerates.

The deposition of agglomerates in the wall becomes more noticeable near the free surface. This is probably due to stratification, with the smallest agglomerates, of higher mobility, preponderant at the very top. After $t \simeq 200 \mathrm{~ms}$ of application of the electric field [Fig. 4(c)] the accumulation of agglomerates on the internal walls of the cell near the splash zone is already significative. Consequently, the intensity of the horizontal flow of agglomerates diminishes in this region. Figure 5 shows in detail the evolution of an agglomerate in the splash zone between $t=117 \mathrm{~ms}$ and $t=311 \mathrm{~ms}$. It is seen how this agglomerate is deflected horizontally until it becomes compacted against the layer of agglomerates already adhered at the wall.

After $t \simeq 300 \mathrm{~ms}$ of application of the electric field [Fig. 4(d)], the layer of agglomerates adhered to the walls becomes appreciable. This region of powder remains in a solidlike state and is neatly distinguishable from the rest of the bed that still remains in a fluidlike state. As the solid layer becomes thicker, some big chunks in the outer region of this layer cannot be supported by the gas flow and fall down in avalanches. At this stage the compaction of powder against the walls starts to produce an appreciable collapse of the free surface of the fluidized region. At the same time, and due to the migration of agglomerates towards the walls, the density of agglomerates in the core region begins to decrease. This creates a less resistance path for the upwards flowing gas. Thus, the local gas velocity increases in the core, which gives rise to an upward flow of agglomerates across this channel.

Figure 4(e) shows the situation after $t \simeq 500 \mathrm{~ms}$ of application of the electric field. At this stage, the solid layer adhered to the walls across the whole bed is significant. A marked upward flow of agglomerates in the core region is observed. When these agglomerates arrive at the free surface, the local gas velocity and therefore the vertical drag force decrease. Then, these aggregates are pushed toward the wall by the horizontal electric field. Downwards avalanches of big aggregates that become detached from the solid layer and cannot be sustained by the gas flow are frequent and the decrease of the fluidized bed height is quite significative.

After $t \simeq 700 \mathrm{~ms}$ [Fig. 4(f)] there are two regions clearly identifiable: the low density core, with an upward flow of agglomerates, and the solid layer adhered to the wall. Both regions are separated by a convective flow of agglomerates, pushed towards the walls by the electric field and falling under the action of gravity when they move to a region of small local gas velocity and become attached to the solid layer to form bigger agglomerates. At this stage the bed height has decreased considerably and the free surface becomes rather diffuse.

Finally, Fig. 4(g) illustrates the stationary state, which is reached after $t \simeq 1 \mathrm{~s}$. The main agglomerate flows are in the vertical direction. Only a small fraction of agglomerates are flowing up across the almost void central channel while a thick solid layer is adhered to the wall, with some agglomerates avalanching down across the interphase between these two regions. The stationary fluidized state is highly heterogeneous and reminds one of the behavior of a spouted bed.

\section{Estimation of electrical charge on the agglomerates}

Since the electric field is almost homogeneous and the effective permittivity of the agglomerates is very small $\left(\epsilon^{* *} \simeq 1.01 \epsilon_{0}\right)$, the physical mechanism behind the deflection of the agglomerates by the electric field must be the action of the electrophoretic force $\left(F_{e} \simeq Q^{* *} E\right)$ due to the presence of an electrostatic charge $Q^{* *}$ on the agglomerates even though the powder as a whole is electroneutral. Using the Stokes' law for an isolated agglomerate, its charge can be estimated as

$$
Q^{* *}=\frac{3 \pi \mu d^{* *} v_{h}^{* *}}{E},
$$

where $v_{h}^{* *}$ is the terminal velocity of the aggregate in the horizontal direction [the time for the agglomerate to reach the terminal velocity is $\tau \sim m^{* *} /\left(3 \pi \mu d^{* *}\right) \sim 1 \mathrm{~ms}$, where $m^{* *}=(1 / 6) \rho_{p} d_{p}^{3}\left(d^{* *} / d_{p}\right)^{D_{a}}$ is the mass of the agglomerate]. In this preliminary investigation we are just concerned with an estimation of the order of magnitude of the charge acquired by the agglomerates in the fluidized state. Uncertainties due to irregular shapes of the aggregates and long range interactions, and the consequent deviations from Stokes' law, are disregarded.

We have analyzed the trajectories of some agglomerates at the initial stage of application of the electric field in the 

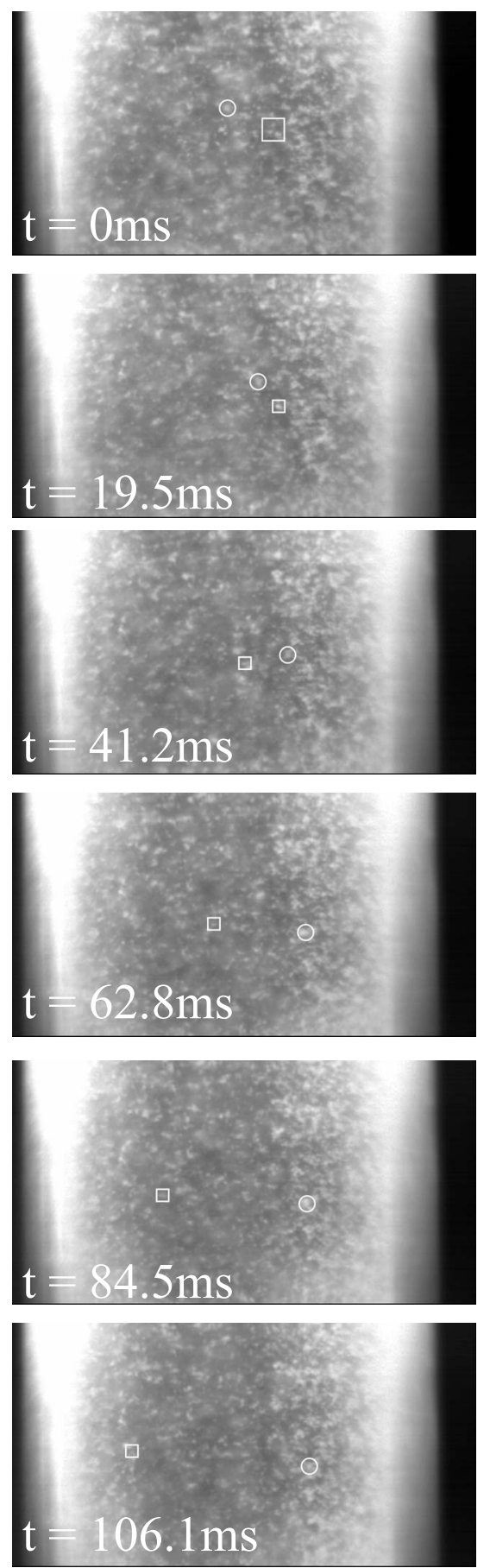

FIG. 6. Snapshots of the fluidized bed near the free surface showing the individual trajectories of two complex agglomerates (squared and circled, respectively). The time elapsed since application of the electric field is indicated. The strength of the applied horizontal field is $E=2.5 \times 10^{5} \mathrm{~V} / \mathrm{m}$. Video of the experiment is available on request.

splash zone, where the density of agglomerates is smaller and flow perturbations are less relevant. The size $d^{* *}$ of each agglomerate has been directly measured as the diameter of the circle with the same area as the agglomerate. Figure 6 shows some snapshots illustrating an example of an almost horizontal trajectory followed by an agglomerate. Between consecutive snapshots there are ten frames, which has allowed us to follow individual trajectories. An additional inconvenient to estimate agglomerate charge stems from the proper agglomeration dynamics of simple agglomerates in the fluidized bed. As seen in Fig. 6, a complex agglomerate may change dynamically and, therefore, its size and shape may be altered during its trajectory towards the wall. Thus, the size and velocity of the agglomerates have been checked in each step of their trajectory.

The motion and size of nanoparticle agglomerates was investigated by manual tracking of the agglomerates. The selection of agglomerates for tracking was based on judgment by the naked eye, avoiding the smaller aggregates in order to minimize the error on size measurement. Since the agglomerate charge should not depend on the strength electric field applied we chose a fixed value of the field strength for the measurements $\left(E=2.5 \times 10^{5} \mathrm{~V} / \mathrm{m}\right)$. Results from this preliminary study are summarized in Table I. The agglomerate charge $Q^{* *}$ of the agglomerates manually tracked was found on the order of $10^{-14} \mathrm{C}$, which gives a charge-to-mass ratio $Q m r$ in the range $10^{-5}-10^{-4} \mathrm{C} / \mathrm{kg}$. This value is similar to the values typically measured for common micrometric or larger particles in high energy operations such as pneumatic transfer [2,3]. In our case, this high value of $Q m r$ can be rationalized from the very high specific surface area of the nanopowder (it is obtained $S_{\text {B.E.T. }} \simeq 1.7 \times 10^{5} \mathrm{~m}^{2} / \mathrm{kg}$ by using the so-called B.E.T. method [11]), which is about 3000 times the specific surface area of powders of $\sim 50 \mu \mathrm{m}$ particles. In fact, if the empirical correlation $Q m r \sim 10^{-7} S$ for powder charging in pneumatic transfer were extrapolated to nanopowders, one would obtain $Q m r \sim 10^{-2} \mathrm{C} / \mathrm{kg}$, which is comparable to the maximum value that $50 \mu \mathrm{m}$ powder particles may acquire at the limit of air breakdown. Our measurements indicate therefore that a low energy operation such as fluidization may charge nanopowders up to quite high levels of $Q m r$ just because of their extremely high specific surface area. This result can be of relevance in the assessment of hazards associated with nanopowder charging [3]. We are currently developing a specific software that will allow us, by means of particle image velocimetry, to obtain robust statistics for deeper insight such as investigating the correlation between agglomerate size and charge.

Assuming that agglomerates can be considered as effective spherical particles, Eq. (2) can be modified to estimate the electrostatic force between agglomerates with charge $Q^{* *}$,

$$
f_{e}^{* *} \sim \frac{\left(Q^{* *}\right)^{2}}{4 \pi \epsilon_{0}\left(d^{* *}\right)^{2}},
$$

where it has been used $\alpha \simeq 0$ and $\beta \simeq 1$ since the agglomerate effective permittivity is close to $\epsilon_{0}$ [35]. If we use $Q^{* *}$ $=10^{-14} \mathrm{C}$ and $d^{* *}=200 \mu \mathrm{m}$, it is estimated $f_{e}^{* *} \sim 0.01 \mathrm{nN}$, which is negligible as compared to the van der Waals force $\left(f_{v d W} \sim 10 \mathrm{nN}\right)$. If it is assumed that the charge $Q^{* *}$ is equidistributed among the simple agglomerates that build up the complex agglomerates, the charge per simple agglomerate would be $Q^{*}=Q^{* *} /\left(d^{* *} / d^{*}\right)^{D_{a}} \sim 10^{-16} \mathrm{C}$, where $d^{*}$ $\sim 30 \mu \mathrm{m}$ and $D_{a} \simeq 2.5$. This charge value gives also a neg- 
TABLE I. Estimation of agglomerate charges using the Stokes' law and measurements of agglomerate size and horizontal terminal velocity during time intervals after application of the electric field.

\begin{tabular}{lccccc}
\hline \hline Time & $\begin{array}{c}\text { Agglomerate } \\
\text { size } \\
\text { interval } \\
(\mathrm{ms})\end{array}$ & $\begin{array}{c}\text { Traveled } \\
\text { distance } \\
(\mathrm{mm})\end{array}$ & $\begin{array}{c}\text { Average } \\
\text { velocity } \\
(\mathrm{cm} / \mathrm{s})\end{array}$ & $\begin{array}{c}\text { Field } \\
\text { strength } \\
(\mathrm{kV} / \mathrm{cm}) \\
\pm 0.3\end{array}$ & $\begin{array}{c}\text { Electric } \\
\text { charge } \\
\left(10^{-14} \mathrm{C}\right) \\
\pm 0.3\end{array}$ \\
\hline $0-9.5$ & 560 & 2.41 & 12.4 & 2.5 & 4.6 \\
$19.5-41.2$ & 600 & 2.27 & 10.5 & 2.5 & 4.3 \\
$41.2-62.8$ & 390 & 1.85 & 8.6 & 2.5 & 2.3 \\
$19.5-41.2$ & 300 & 2.27 & 10.5 & 2.5 & 2.1 \\
$41.2-62.8$ & 370 & 2.56 & 11.9 & 2.5 & 2.9 \\
$62.8-84.5$ & 370 & 3.98 & 18.3 & 2.5 & 4.5 \\
$84.5-106.1$ & 250 & 2.41 & 11.2 & 2.5 & 1.9 \\
$117.0-138.6$ & 490 & 1.07 & 4.6 & 2.5 & 1.5 \\
$138.6-160.3$ & 480 & 1.14 & 5.3 & 2.5 & 1.7 \\
$160.3-181.9$ & 450 & 1.14 & 5.3 & 2.5 & 1.6 \\
$181.9-203.6$ & 450 & 1.70 & 7.8 & 2.5 & 2.4 \\
$203.6-225.3$ & 530 & 1.56 & 7.2 & 2.5 & 2.6 \\
$225.3-246.9$ & 580 & 1.42 & 6.6 & 2.5 & 2.6 \\
$246.9-268.6$ & 520 & 1.56 & 7.2 & 2.5 & 2.5 \\
$268.6-290.2$ & 530 & 1.42 & 6.6 & 2.5 & 2.4 \\
$290.2-311.9$ & 530 & 0.99 & 4.6 & 2.5 & 1.6 \\
\hline \hline
\end{tabular}

ligible electrostatic force between simple agglomerates $\left(f_{e}^{*}\right.$ $\left.\sim 10^{-4} \mathrm{nN}\right)$. From these estimations, it can be concluded that in the absence of an external electric field, the electrostatic forces do not play a role in the agglomeration of nanoparticles in the fluidized bed.

The agglomerates charged with the same sign are compacted against the wall with a force $f_{c} \sim Q^{* *} E \sim 1-10 \mathrm{nN}$, which is larger than their own weight $\left[w^{* *}=w_{p}\left(d^{* *} / d_{p}\right)^{D_{a}}\right.$ $\sim 1 \mathrm{nN}]$, and is similar to the van der Waals attractive force in the absence of compaction. It is well known that van der Waals attractive forces between agglomerates of fine particles are enhanced by compaction [51] while as we have seen the electrostatic forces, either attractive or repulsive, are negligible. Thus, it is explainable that the cohesiveness of the layer of agglomerates is increased by compaction and it remains adhered to the wall even when the field is turned off as seen experimentally.

\section{CONCLUSIONS}

We have analyzed the effect of an electrostatic field horizontally applied on the behavior of a fluidized bed of nanopowders. A main result from this analysis is that nanoparticle agglomerates carry an electrostatic charge in the fluidized bed of a value of the order of $10^{-14} \mathrm{C}$, which is comparable to the typical charge measured in pneumatic conveying of micron sized and larger particles. This high charge level acquired in fluidization is attributable to the large spe- cific surface area of nanopowders. This work provides for the first time an estimation of the agglomerate charge in fluidization that can be useful in the assessment of potential hazards in industry operations.

The electrostatic forces between agglomerates due to the estimated charge are negligible as compared to the attractive van der Waals force. Thus, the charge of nanoparticles in the fluidized bed does not have an influence on the agglomeration dynamics. When a strong electric field is applied, neither the forces between induced dipoles are significant to affect the agglomerate size or structure. Instead, the action of the field is to push the agglomerates towards the lateral walls of the bed. The appearance of the bed after application of the electric field in the stationary state is reminiscent of a spouted bed, with a solidlike layer of powder adhered to the walls and a central low density channel through which most of the gas bypasses the powder. As a consequence, bed expansion is hindered and the uniform fluidization state turns to a highly heterogeneous fluidization state when the electrostatic field is applied.

\section{ACKNOWLEDGMENTS}

We acknowledge Spanish Government Agency Ministerio de Ciencia y Tecnologia (Contract No. FIS2006-03645) and Junta de Andalucia (Contract No. FQM 421). We thank Daniel Lepek, from the New Jersey Institute of Technology, who helped us in performing the bed expansion experiments during his stay at the University of Seville. 
[1] H. F. Eden, in Electrostatic Nuisances and Hazards, edited by A. D. Moore, Electrostatics and Its Applications (Wiley, New York, 1973), pp. 425-440.

[2] M. Glor, J. Electrost. 16, 175 (1985).

[3] M. Glor, Electrostatic Hazards in Powder Handling (Wiley, New York, 1988), pp. 425-440.

[4] A. G. Bailey, J. Electrost. 30, 167 (1993).

[5] H. Mizes, M. Ott, E. Eklund, and D. Hays, Colloids Surf., A 165, $11(2000)$.

[6] R. J. Han, O. R. Moss, and B. A. Wong, Aerosol Sci. Technol. 21, 241 (1994).

[7] J. D. Cobine, in Other electrostatic effects and applications, edited by A. D. Moore, Electrostatics and Its Applications (Wiley, New York, 1973), pp. 441-455.

[8] J. E. Lawver and W. P. Dyrenforth, in Electrostatic Separation, edited by A. D. Moore, Electrostatics and Its Applications (Wiley, New York, 1973), pp. 221-249.

[9] M. Yuji, J. Soc. Powder Technol. Jpn. 43, 205 (2006).

[10] C. Nam, R. Pfeffer, R. N. Dave, and S. Sundaresan, AIChE J. 50, 1776 (2004).

[11] C. Zhu, Q. Yu, R. N. Dave, and R. Pfeffer, AIChE J. 51, 426 (2005).

[12] X. S. Wang, V. Palero, J. Soria, and M. J. Rhodes, Chem. Eng. Sci. 61, 5476 (2006).

[13] X. S. Wang, V. Palero, J. Soria, and M. J. Rhodes, Chem. Eng. Sci. 61, 8040 (2006).

[14] J. M. Valverde, M. A. S. Quintanilla, A. Castellanos, D. Lepek, J. Quevedo, R. N. Dave, and R. Pfeffer, AIChE J. 54, 86 (2008).

[15] W. Yao, G. Guangsheng, W. Fei, and J. Wu, Powder Technol. 124, 152 (2002).

[16] L. F. Hakim, J. L. Portman, M. D. Casper, and A. W. Weimer, Powder Technol. 160, 149 (2005).

[17] S. Matsuda, et al., AIChE J. 50, 2763 (2004).

[18] C. Zhu et al., Powder Technol. 141, 119 (2004).

[19] Q. Yu et al., AIChE J. 51, 1971 (2005).

[20] J. Quevedo et al., AIChE J. 52, 2401 (2006).

[21] J. M. Valverde and A. Castellanos, Chem. Eng. Sci. 62, 6947 (2007).

[22] M. Kashyap, D. Gidaspow, and M. Driscoll, Powder Technol. (to be published).

[23] H. A. Pohl, in Nonuniform Field Effects: Dielectrophoresis, edited by A. D. Moore, Electrostatics and Its Applications (Wiley, New York, 1973), pp. 336-362.

[24] F. Sachsse, Ann. Phys. 14, 396 (1932).

[25] W. B. Kunkel and J. W. Hansen, Rev. Sci. Instrum. 21, 308 (1950).

[26] W. B. Kunkel, J. Appl. Phys. 21, 820 (1950).

[27] S. Matsusaka and H. Masuda, Adv. Powder Technol. 14, 143
(2003).

[28] D. Hochrainer, Ann. Occup. Hyg. 29, 241 (1985).

[29] H. Y. Wen, G. P. Reischl, and G. Kasper, J. Aerosol Sci. 15, 103 (1984).

[30] J. M. Valverde and A. Castellanos, AIChE J. 52, 838 (2006).

[31] L. B. Loeb, Static Electrification (Springer, Berlin, 1958), p. 166.

[32] G. S. P. Castle, J. Electrost. 40\&41, 13 (1997).

[33] I. I. Inculet, in Static Electrification of Dielectrics and at Materials' Interfaces, edited by A. D. Moore, Electrostatics and Its Applications (Wiley, New York, 1973), pp. 86-114.

[34] C. D. Hendricks, in Electrostatic Imaging, edited by A. D. Moore, Electrostatics and Its Applications (Wiley, New York, 1973), pp. 281-306.

[35] J. Q. Feng, Phys. Rev. E 62, 2891 (2000).

[36] J. Q. Feng and D. A. Hays, Powder Technol. 135\&136, 65 (2003).

[37] ASM Engineered Materials Reference Book, 2nd ed., edited by Michael Bauccio (ASM International, Materials Park, OH, 1994); http://www.matweb.com

[38] D. A. Hays, in Adhesion of Charged Particles, edited by D. S. Rimai, L. P. DeMejo, and K. L. Mittal, Fundamentals of Adhesion and Interfaces (VSP Utrecht, The Netherlands, 1995) pp. 61-71.

[39] B. Gady, D. Schleef, R. Reifenberger, D. Rimai, and L. P. DeMejo, Phys. Rev. B 53, 8065 (1996).

[40] H. C. Hamaker, Physica (Amsterdam) 4, 1058 (1937).

[41] J. Visser, Adv. Colloid Interface Sci. 3, 331 (1972).

[42] H. Krupp, Adv. Colloid Interface Sci. 1, 111 (1967).

[43] L. Massimilla and G. Donsi, Powder Technol. 15, 253 (1976).

[44] K. Rietema, The Dynamics of Fine Powders (Elsevier, London, 1991).

[45] D. M. Schaeffer, M. Carpenter, B. Gady, R. Reifenberger, L. P. DeMejo, and D. S. Rimai, in Surface roughness and its influence on particle adhesion using atomic force techniques, edited by D. S. Rimai, L. P. DeMejo, and K. L. Mittal, Fundamentals of Adhesion and Interfaces (VSP, Utrecht, The Netherlands, 1995) pp. 35-48.

[46] S. T. Nase, W. L. Vargas, A. A. Abatan, and J. J. McCarthy, Powder Technol. 116, 214 (2001).

[47] W. Wen and K. Lu, Phys. Rev. E 55, R2100 (1997).

[48] M. J. Espin, A. V. Delgado, and S. Ahualli, IEEE Trans. Dielectr. Electr. Insul. 13, 462 (2006).

[49] J. D. Jackson, Classical Electrodynamics (Wiley, New York, 1999).

[50] K. Karkkainen, A. Sihvola, and K. Nikoskinen, IEEE Trans. Geosci. Remote Sens. 39, 1013 (2001).

[51] M. A. S. Quintanilla, J. M. Valverde, and A. Castellanos, AIChE J. 52, 1715 (2006). 